\title{
Relationships between the AE, ap and Dst indices near solar minimum (1974) and at solar maximum (1979)
}

\author{
M. M. Fares Saba, W. D. Gonzalez, A. L. Clúa de Gonzalez \\ Instituto Nacional de Pesquisas Espaciais, Av. dos Astronautas, 1758 - Cx. Postal 515, 12227-010 - São José dos Campos, SP, Brazil
}

Received: 18 September 1995 / Revised: 9 April 1997 / Accepted: 29 April 1997

\begin{abstract}
Three-hourly average values of the Dst, AE and ap geomagnetic activity indices have been studied for 1 year's duration near the solar minimum (1974) and also at the solar maximum (1979). In 1979 seven intense geomagnetic storms (Dst $<-100 \mathrm{nT}$ ) occurred, whereas in 1974 only three were reported. This study reveals: (1) the yearly average of AE is greater in 1974 than in 1979, whereas the inverse seems to be true for the yearly average of Dst, when a higher number of intense storms is present. These averages indicate the kind of activity occurring on the sun as shown in earlier work. (2) The seasonal variation of Dst is higher than that of ap and is almost negligible in AE. (3) The correlation coefficient of ap $\times \mathrm{AE}$ is in general the highest, as the magnetometers that monitor both indices are close, and is surpassed only by the ap $\times$ Dst correlation during geomagnetic storms, when the influence of the ring current is dominant. The correlation of ap $\times$ Dst also shows a seasonal variability. (4) For the first time a study of correlation between ap and a linear combination of AE and Dst has also been made. We found higher correlation coefficients in this case as compared to those between ap $\times$ Dst and ap $\times$ AE.
\end{abstract}

\section{Introduction}

In the study of solar-terrestrial relationships, the use of indices of geomagnetic activity plays an important role. The four most commonly used indices are AE, Dst, Kp and the linear counterpart of the latter, ap.

Available since 1932, the Kp index represents the intensity of planetary magnetic activity as seen at subauroral latitudes and is given for each 3-h interval. The individual $\mathrm{K}$ indices for each of the contributing

Correspondence to: M. M. Fares Saba mid-latitude observatories reflect the maximum range of any component of the field over the 3-h interval at each station. The $\mathrm{Kp}$ index is the average of the $\mathrm{K}$ values from all contributing observatories. A conversion scale transforms the quasi-logarithmic Kp to a linear index named ap.

AE was defined in 1966 by Davis and Sugiura so that it measures primarily the variations in the auroral electrojets. It is based on 1-min values of the $\mathrm{H}$ component trace from auroral-zone observatories located around the world. The data of these observatories are plotted as a function of universal time. The upper and lower envelopes are defined as AU and AL indices, respectively, and are believed to represent the maximum eastward and westward electrojet currents. The sum of the absolute values of AL and AU is called AE.

Introduced in 1964, the ring-current index Dst measures primarily the ring-current magnetic field. It is based on hourly averages of the $\mathrm{H}$ component recorded at four low-latitude observatories, subtracting the average $\mathrm{Sq}$ and the permanent field from the disturbed magnetic field. A detailed description of the methods by which these indices are derived and the correspondent uncertainties can be found in Mayaud (1980).

Although these three indices have been calculated for many years, only a few studies on their interrelationships have been published (Campbell, 1979; Rostoker, 1991; Cade et al., 1995). The purpose of this study is to understand better the possible relationships between them. Further, this study was done for two different epochs of the same solar cycle. During the descending phase of the solar cycle, near solar minimum (1974), it is known that stable coronal holes on the sun lead to the existence of recurrent high-speed streams that are observed at earth during every solar rotation. On the other hand, at solar maximum (1979), when large-scale coronal holes retract back to higher latitudes, what produces the observed high-speed streams responsible for the storms are transient solar phenomena generally known as coronal mass ejections (involving the presence of flares or filament eruptions). 
Besides comparing several types of averages of these indices in pairs, in this paper we have attempted for the first time to compare averages of the ap index with a linear combination of the AE and Dst indices. A physical basis for this attempt refers to the fact that processes occurring at high and low latitudes, as monitored by the AE and Dst indices, respectively, are expected to influence equally the mid-latitudes where the ap index is recorded.

\section{Methods of data analysis}

For convenience, in this work we have used ap instead of $\mathrm{Kp}$, since ap is based on a linear scale, whereas $\mathrm{Kp}$ is on a quasi-logarithmic scale.

Since ap is a 3 -h index, Dst and AE were averaged for the same interval for the purpose of comparison. This implies that the results of this study have a resolution limited to a 3-h interval and therefore higher-resolution phenomena like substorms could not be studied. Figure 1 shows an example of three-hourly values of Dst, ap and AE. Some correlation (or anticorrelation) between them is easily seen.

In order to understand how these indices respond to interplanetary sources of activity during different periods of the solar cycle, annual and monthly averages were studied for the two years of interest, 1974 and 1979.

A physical relationship between two different indices can be estimated quantitatively by the Pearson correlation method, calculating the linear correlation coefficient $r$. This coefficient indicates whether or not the two indices are related. To investigate the correlation
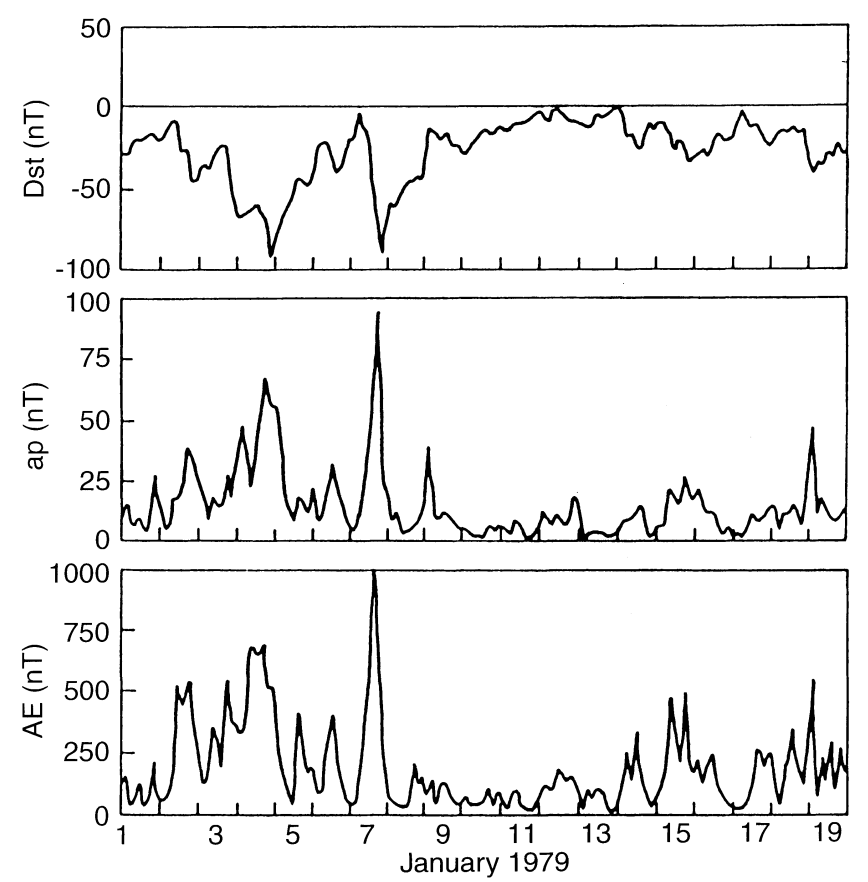

Fig. 1. Example of three-hourly average values of Dst, ap and AE for the interval of 1-19 January 1979 between one index and two others, a multiple correlation coefficient was calculated. This coefficient serves as a useful tool for testing whether one particular index can be expressed in terms of other two. In particular, several multiple correlations were done to find how ap could be expressed in terms of AE and Dst for different timeintervals and geomagnetic conditions. We use the following expressions for these two methods, as described by Bevington (1969).

The linear adjust is given by:

$y=a+\sum_{j=1}^{n} b_{j} x_{j}$,

where in this paper $y$ and $x_{j}, j=1,2(n=2)$, represent the different geomagnetic indices considered for multiple correlation, and $a$ and $b_{j}$ are the coefficients obtained from the best fit.

The sample covariance $s_{j k}^{2}$ is:

$s_{j k}^{2} \equiv \frac{\frac{1}{N-1} \sum_{i=1}^{N}\left[\frac{1}{\sigma_{i}^{2}}\left(x_{i j}-\bar{x}_{j}\right)\left(x_{i k}-\bar{x}_{k}\right)\right]}{\frac{1}{N} \sum_{i=1}^{n}\left(1 / \sigma_{i}^{2}\right)}$,

where $j, k=1,2 ; \sigma_{i}$ is the standard deviation, $N$ is the number of data points and $\bar{x}_{j}$ is

$\bar{x}_{j} \equiv \frac{\sum_{i=1}^{N}\left(x_{i j} / \sigma_{i}^{2}\right)}{\sum_{i=1}^{N}\left(1 / \sigma_{1}^{2}\right)}$.

The sample variance is given by:

$s_{j}^{2} \equiv s_{j j}^{2}$.

The linear-correlation coefficient can be expressed in terms of:

$r_{j k} \equiv \frac{s_{j k}^{2}}{s_{j} s_{k}}$.

In analogy with this definition of the linear-correlation coefficient, we define the multiple-correlation coefficient $R$ to be:

$$
R^{2} \equiv \sum_{j=1}^{n}\left(b_{j} \frac{s_{j y}^{2}}{s_{y}^{2}}\right)=\sum_{j=1}^{n}\left(b_{j} \frac{s_{j}}{s_{y}} r_{j y}\right) \text {. }
$$

\section{Results}

\subsection{Average values}

3.1.1 Annual averages. In Fig. 2 one can see that the annual average of AE for 1974 (dashed line across the bar graph) is greater than that for 1979 (solid line). This indicates a greater intensity of the auroral eletrojects in 1974. Presumably the recurrent streams strongly present during this year can be thought of as being responsible for the higher occurrence of substorms. These recurrent 

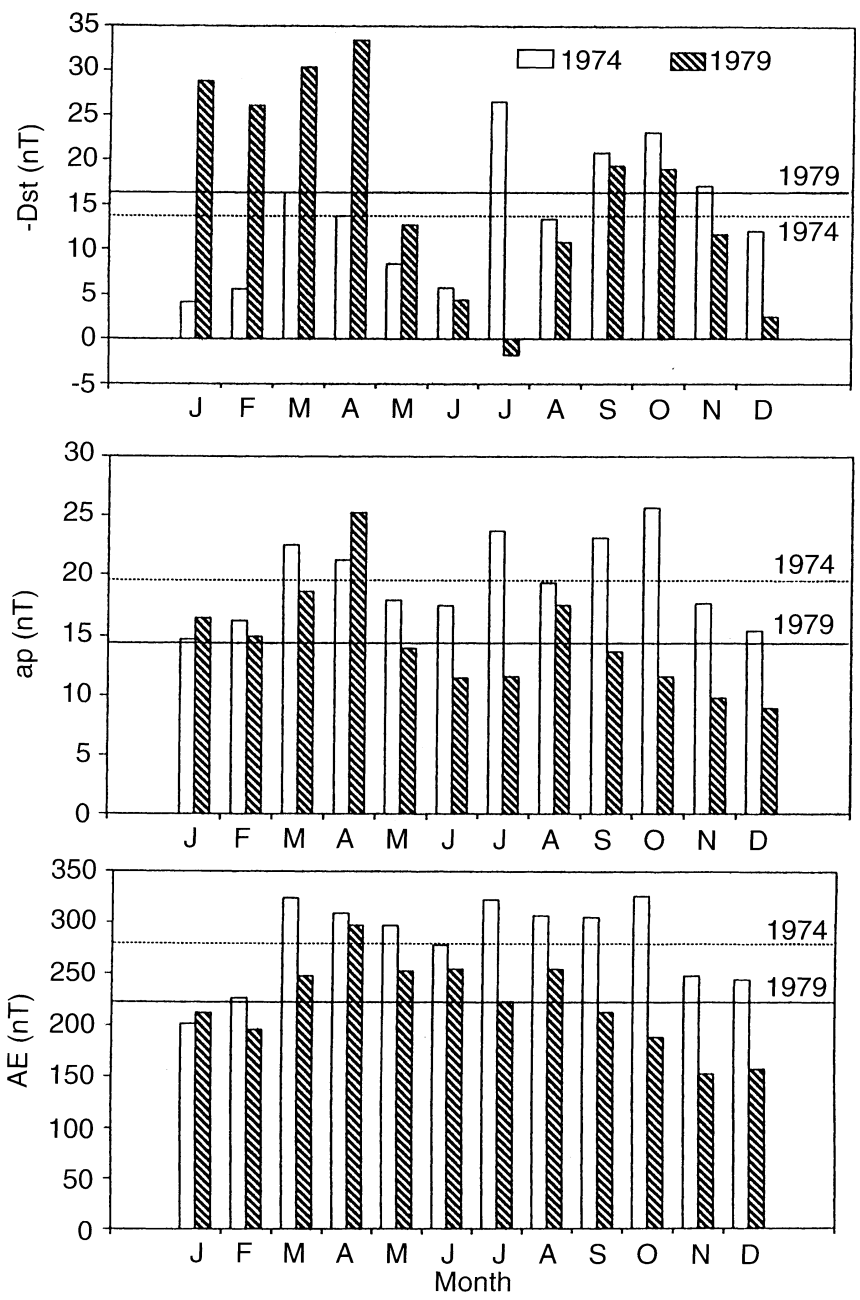

Fig. 2. Monthly averages of AE, ap and -Dst; the annual averages are marked with solid (1979) and dashed (1974) horizontal lines

streams have their origin in coronal holes frequently observed near solar minimum periods. They cause more substorms due to the large amount of alfvénic fluctuations, wherein $B_{z}$ fields do not last long enough to influence substantially the ring current and therefore produce storms. On the other hand, solar transients are expected to cause storms when the $B_{z}$ fields associated with the related interplanetary structures are intense enough and are of long duration. In the latter case the occurrence of substorms is mainly restricted to the main phase of the storms and does not last as long (hours) as the Alfvén wave trains (days) of corotating streams (Tsurutani et al., 1995). Besides this interpretation, it is possible that near solar maximum, during intense storms, the auroral oval grows to lower latitudes and the $\mathrm{AE}$ chain of magnetometers cannot monitor the auroral electroject activity properly (Feldstein, 1992). This could also contribute to a lower average of AE near solar maximum.

On the other hand, 1979 is characterized by intense solar transients (Tsurutani et al., 1988) and shows higher intensities in the ring current and consequently a higher occurrence of geomagnetic storms. As a result, the average of Dst for 1979 is greater than the average for
1974. The average of ap is higher for 1974 , following the trend of AE to which it is, in general, better correlated, as will be seen.

3.1.2 Monthly averages. The seasonal variation of geomagnetic activity has been known to be associated with an enhancement in geomagnetic activity at the equinoxes (Russell and McPherron, 1973), and are thought to be due mainly to an effective coupling of the interplanetary magnetic field with the earth's geomagnetic field.

Figure 2 shows that this seasonal variation of geomagnetic activity (Clua de Gonzalez et al., 1993) is present in the monthly averaged indices for 1974 and 1979. However, this variability is higher for Dst than that for ap, and is hardly noticed in AE. An anomalous seasonal peak was also found in July 1974 and is probably similar to that reported by Clua de Gonzalez et al. (1993). No explanation however, has been proposed for that anomalous peak until now.

\subsection{Correlations between two indices}

3.2.1 Lags in the correlations. Figure 3 shows correlation curves for pairs of indices among AE, ap and Dst, both for 1974 and 1979. A finite time-lag was observed in the
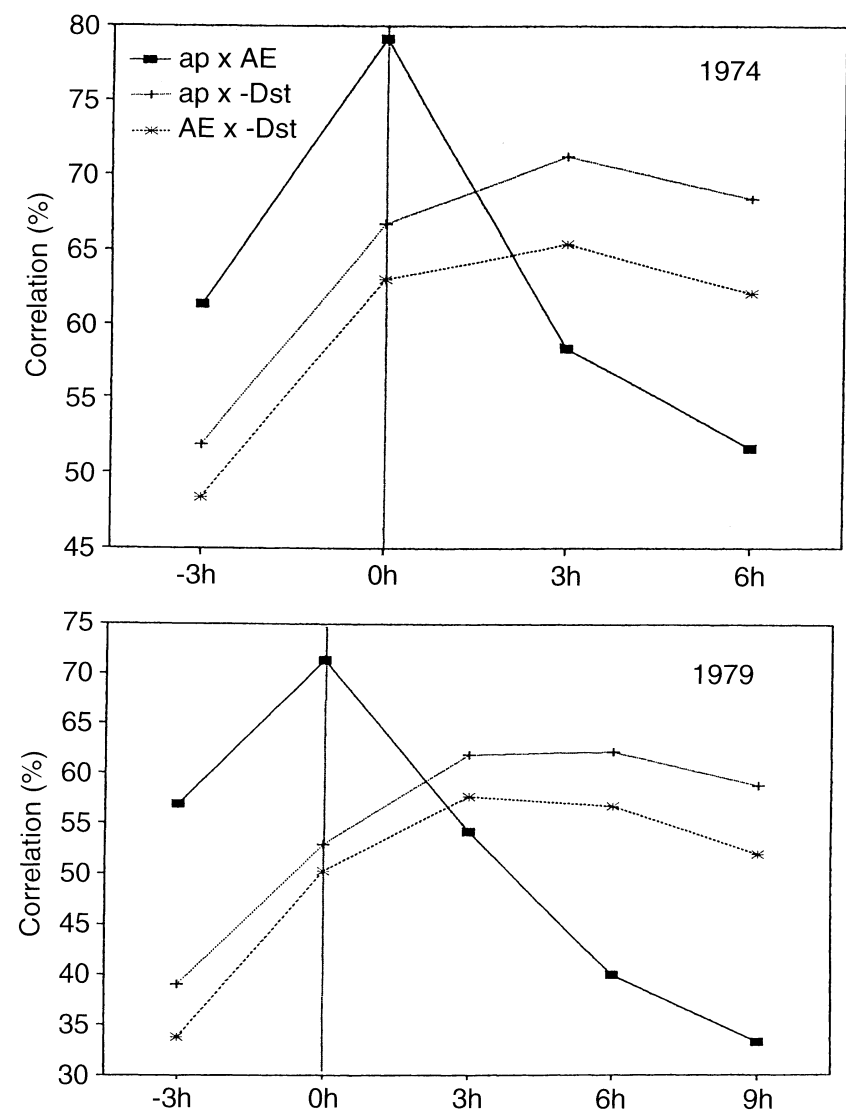

Fig. 3. Time-lag for annual correlations; the numbers in the $x$-axis represent the time-lag of the second index in relation to the first 
correlations between Dst and AE and between Dst and ap, but no time-lag was found when correlating AE with ap. The latter is probably due to the similar nature of the currents which are monitored by these indices. On the other hand, while Dst monitors the ring current (a bulky and low-rate dissipating current), the ap and AE respond to ionospheric currents with a faster response time to external variations.

The time-lag was found to be around $1 \mathrm{~h}$ for correlations between hourly values of $\mathrm{AE}$ and Dst. Since the resolution of ap is $3 \mathrm{~h}$, all correlations presented in this work are calculated for three-hourly values. In such a case, the time-lags for correlations of $\mathrm{AE}$ versus Dst and ap versus Dst are about $3 \mathrm{~h}$, or one correlation interval. Due to this broad time resolution, the actual lag would lie between 0 and $3 \mathrm{~h}$.

3.2.2 Annual correlations. Figure 4 shows the three possible annual correlations between ap, AE and Dst for 1974 and 1979. Above each bar we show the correlation coefficient and its time-lag is given above that. One can see that in 1974 the correlations are higher than in 1979, possibly because recurrent streams make the geomagnetic activity more constant along 1974 and not sporadic as in 1979. Further, the correlation between ap and AE is the highest of all, as they both monitor similar ionospheric currents and have their geomagnetic stations fairly close. Not so well correlated are ap and Dst, since Dst monitors mainly the ring current, whereas ap responds strongly to ionospheric currents as well. However, it was also observed that during geomagnetic storm periods ap is better correlated to Dst. On the other hand, although AE and Dst are poorly correlated, they still seem to have some relationship with each other, since both are expected to respond to common energization processes.

3.2.3 Monthly correlations. For all months of 1974 and 1979 the three preceding correlations were calculated. Their correlation coefficients were found to be similar to those obtained for the annual correlation. However, it

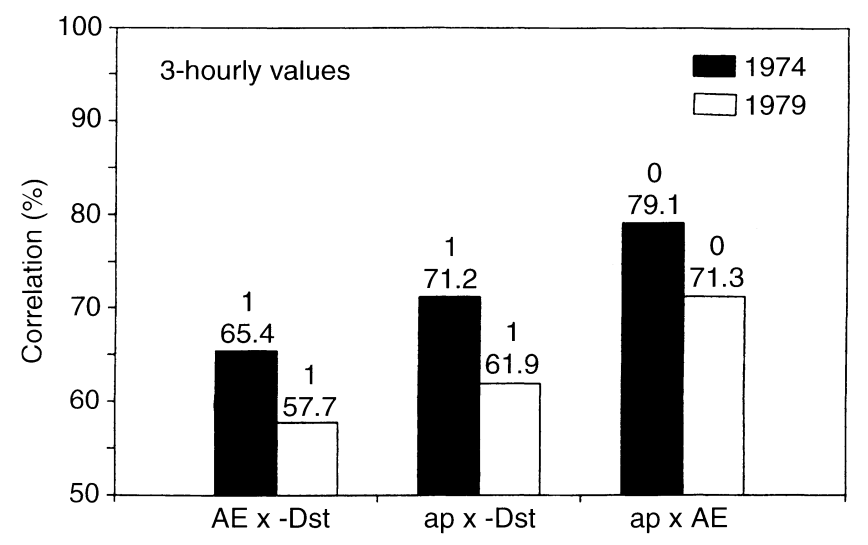

Fig. 4. Annual correlations; the lag of the best correlation for each case is shown on the top of each column was found that the ap $\times$-Dst correlation is highly influenced by the level of activity in the ring current, which is mainly measured by Dst, as shown in Fig. 5. The monthly variability of Dst is also shown for comparison here. Figure 5 also shows a seasonal variability in the ap $\times$-Dst correlation, probably due to the seasonal variations of ap and especially of Dst, as shown in Fig. 2. The anomalous seasonal peak of Dst in July 1974, already mentioned, causes a much better correlation with ap during this month, and thus a peak is also observed in the monthly correlation of ap $\times-$ Dst for 1974.

3.2.4 Correlations during geomagnetic storms. Geomagnetic storms can be divided into three phases: initial, main and recovery phases. The initial phase may be gradual, or be represented by an abrupt change in the Dst, called a sudden commencement. The main phase of a storm is defined to begin when the Dst assumes negative values and ends when it reaches its minimum decrease. The recovery phase, usually the longest one, is characterized by the returning of Dst to its pre-sudden commencement values.

In order to study the correlations between AE, Dst and ap during storm periods, seven intense $\left(\right.$ Dst $\left._{\min }<-100 \mathrm{nT}\right)$ storms and eleven moderate $\left(-100 \mathrm{nT}<\mathrm{Dst}_{\min }<-75 \mathrm{nT}\right)$ storms were chosen from 1979 (Gonzalez et al., 1989; Mendes, 1992). Figure 6 shows the correlations between pairs of the AE, ap and Dst indices during the main and recovery phases of the storm. The annual correlations are also shown for comparison.

One can notice in this figure that the correlations are higher during storm periods than during more extended

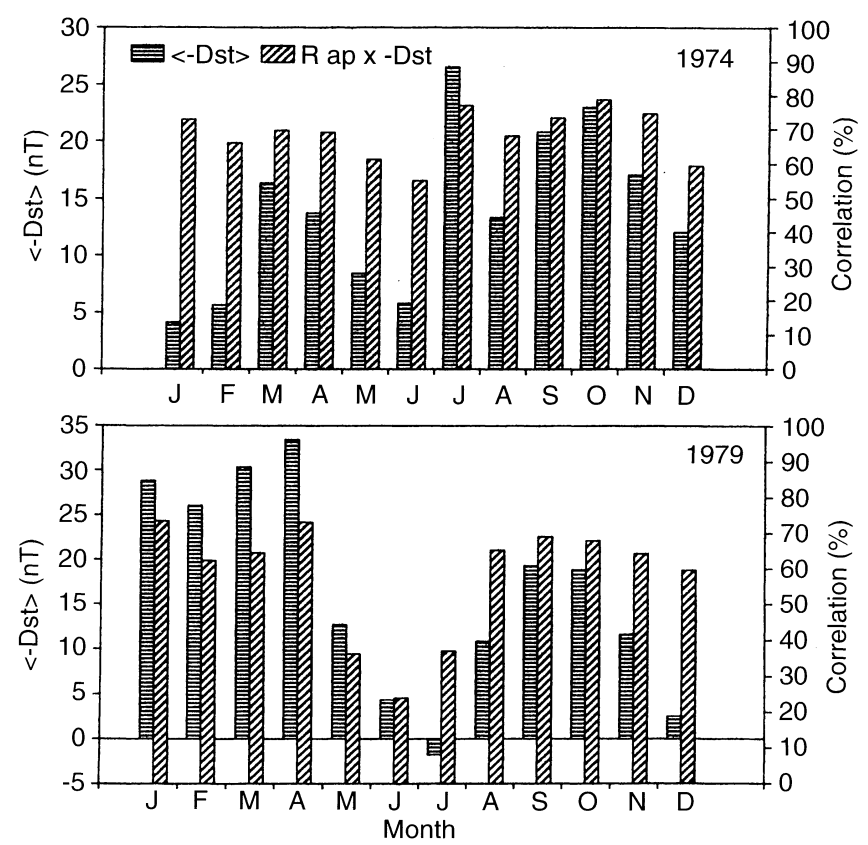

Fig. 5. Monthly average values of Dst and the monthly correlations of ap $\times$-Dst for 1974 and 1979 


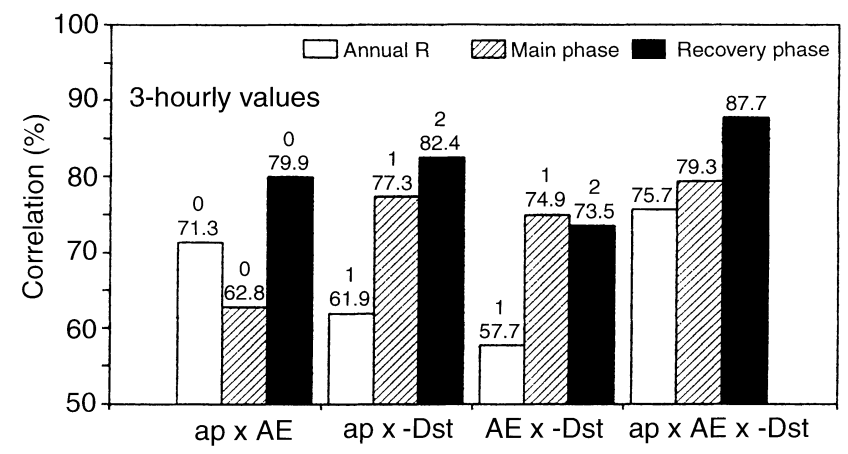

Fig. 6. Annual correlations and correlations during magnetic storm phases for 1979; Multiple correlation between ap, AE and Dst for the same period is shown in the last group. The lag of the best correlation for each case is shown on the top of each column

periods, since all indices reveal an increase with higher magnetosphere activity. However, the ap $\times$ AE correlation is surprisingly low in the main phase period. This is probably caused by the higher influence of the intense ring current over the ap index and because $\mathrm{AE}$ is fluctuating faster and more intensively when the auroral electrojects vary during this phase. Furthermore, the intensification of the electrojects may cause the auroral oval to expand equatorwards during major geomagnetic storms, so that the AE stations would not be able to monitor them properly. The difference in time resolution of these two indices could also be another possible explanation for this discrepancy. It may be possible that the shorter time-scale effects are somehow transferred better to the computed averages of Dst and AE values, while ap is by definition a slow index. Then the correlation between AE and Dst would be good, although the correlation between $\mathrm{AE}$ and ap is poor. This effect could decrease the correlation between ap and AE more than between ap and Dst, because Dst is slower than AE.

In the recovery phase, the energy injection, which produces intense perturbations during the main phase, is nearly non-existent, with a consequent recovery of all indices, and therefore all correlations tend to increase. However, since the dissipation of the auroral currents is faster than for the ring current, the corresponding timelags between Dst and the other two indices tend to increase, whereas the correlation between Dst and AE tends to decrease.

\subsection{Multiple correlations}

The ap index is well correlated with Dst and even more with AE, as seen before. It also has an intermediate behaviour as noticed by Rostoker (1972), who found that "although the Kp index is capable of describing the general state of planetary geomagnetic activity, it contains contributions from at least two major sources, the auroral eletroject and the ring current". By means of a multiple correlation program a linear fit was performed for ap in terms of AE and Dst, namely:

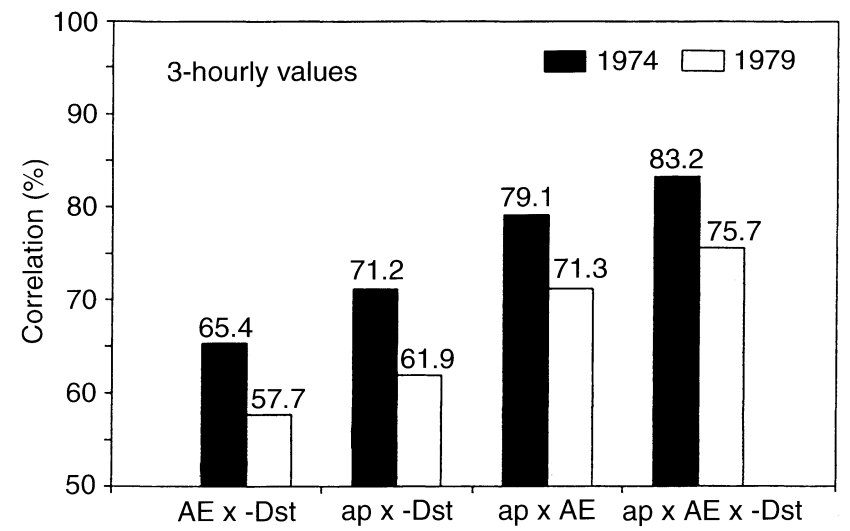

Fig. 7. Annual correlations and multiple correlation for 1974 and 1979

$\mathrm{ap}=a+b \mathrm{AE}+c(-\mathrm{Dst})$

expressing, therefore, quantitatively what was only qualitatively expected.

For these linear fits Dst is lagged by one interval (0$3 \mathrm{~h}$ ) in relation to ap and $\mathrm{AE}$, since this was the most common time-lag found in the previous correlations.

3.3.1 Annual multiple correlations. For the years 1974 and 1979 the following correlation coefficients, linear fit coefficients and errors (inside parenthesis) were obtained:

\begin{tabular}{lllll}
\hline Year & $\begin{array}{l}\text { Correlation } \\
\text { coefficient (\%) }\end{array}$ & $a$ & $b\left(\times 10^{-2}\right)$ & $c\left(\times 10^{-2}\right)$ \\
\hline 1974 & 83.2 & $-1.10(0.05)$ & $5.5(0.1)$ & $36.6(1.5)$ \\
1979 & 75.7 & $-0.60(0.04)$ & $5.1(0.1)$ & $23.0(1.1)$ \\
\hline
\end{tabular}

Figure 7 shows a comparison between the pairs of annual correlations presented and those of the multiple correlation. The latter are clearly higher both in 1974 and in 1979. The constant terms of the fittings $(a)$ are relatively low, showing that the main sources that contribute to ap are the auroral electrojects and the ring current.

3.3.2 Multiple correlation for monthly values. For longer periods of analysis one could use monthly averages of the indices instead of three-hourly values. The correlation coefficients and the linear fit coefficients for 1974 and 1979 are:

\begin{tabular}{llrll}
\hline Year & $\begin{array}{l}\text { Correlation } \\
\text { coefficient }(\%)\end{array}$ & \multicolumn{1}{l}{$l$} & $b\left(\times 10^{-2}\right)$ & $c\left(\times 10^{-2}\right)$ \\
\hline 1974 & 94.1 & $3.3(1.3)$ & $4.6(1.3)$ & $23.6(7.7)$ \\
1979 & 94.4 & $-2.9(1.6)$ & $6.1(1.2)$ & $23.1(4.7)$ \\
\hline
\end{tabular}

3.3.3 Multiple correlation during geomagnetic storms. Multiple correlations were also done for the geomagnetic data obtained during the same seven intense and 
eleven moderate storms selected to study simple pairs of correlations (Sect. 3.2.4). The correlation coefficients and the linear fits for the main and recovery phases of these storms are:

\begin{tabular}{lllll}
\hline Phase & $\begin{array}{l}\text { Correlation } \\
\text { coefficient }(\%)\end{array}$ & $a$ & $b\left(\times 10^{-2}\right)$ & $c\left(\times 10^{-2}\right)$ \\
\hline main & 79.3 & $-15.8(26.2)$ & $4.5(1.4)$ & $66(10)$ \\
recovery & 87.7 & $-13.7(16.0)$ & $6.6(0.5)$ & $42(4)$ \\
\hline
\end{tabular}

Note that the coefficient $a$ has increased with respect to the previous two multiple correlation cases, since the ap values involved in the latter case are higher. Figure 6 shows the multiple correlation for the whole year 1979 and for the two phases of the selected storms.

\section{Conclusions}

In this work an effort has been made to achieve a better understanding of the geomagnetic indices and their relationships during two different periods of the solar cycle by means of indices, their averages and correlations. The main conclusions can be summarized as follows.

The annual average of AE observed in 1974 (near solar minimum) is greater than in 1979 (solar maximum), whereas average Dst is greater in 1979, a year characterized by intense solar transients. The higher occurrence of substorms is responsible for a higher AE, whereas the occurrence of several storms gives a higher Dst for 1979. The reason for this was summarized in Sect. 4.

The seasonal variation associated with an enhancement in geomagnetic activity at the equinoxes was found to be highest for Dst. However, it can be also seen in the ap distribution, but hardly noticed in AE.

The correlation coefficient of ap $\times$ AE (three-hourly values, lag $0, r=79.1 \%$ ) is in general the highest of all, being surpassed only by that of ap $\times$-Dst (three-hourly values, lag $2, r=82.4 \%$ ) during geomagnetic storms; ap $\times$-Dst shows a clear seasonal variation in a similar manner to the Dst index itself. A finite time-lag was observed in the correlations between Dst and AE and between Dst and ap, but no lag was found when correlating AE with ap, as both indices mainly monitor ionospheric electrojects.

Finally, for the first time, a multiple correlation study among the three indices was made with three-hourly values of these indices, annually for 1974 and 1979, for monthly averages and for geomagnetic storm periods. It was found that a better correlation is achieved when correlating all three indices and that Dst has a greater influence on ap during periods of higher ring-current activity (geomagnetic storms). We believe that these relationships would be useful in estimation of one index from the other two (known indices). Also, one could estimate AE values prior to 1966 in terms of ap and Dst for the period for which $\mathrm{AE}$ values have not been reported; or even fill gaps that accidentally appear in the data bank of one of these indices.

Acknowledgements. This work was partially supported by the Fundação de Amparo à Pesquisa do Estado de São PauloFAPESP and by the Fundo Nacional de Desenvolvimento Científico e Tecnológico of Brazil. The AE, Dst and ap data used in this study were obtained from magnetic tapes provided by the World Data Center A in Boulder, Colorado.

Topical Editor K.-H. Glaßmeier thanks T. Araki and K. Kauristie for their help in evaluating this paper.

\section{References}

Bevington, P. R., Data reduction and error analysis for the physical sciences, McGraw-Hill, New York, 1969.

Cade III, W. B., J. J. Sojka, and L. Zhu, A correlative comparison of the ring current and auroral electrojects using geomagnetic indices, J. Geophys. Res., 100, 97-105, 1995.

Campbell, W. H., Occurrence of AE and Dst geomagnetic index levels and the selection of the quietest days in a year, J. Geophys. Res., 84, 875-881, 1979.

Clua de Gonzalez, A. L., W. D. Gonzalez, and S. L. G. Dutra, Periodic variations in geomagnetic activity: a study based on the Ap index, J. Geophys. Res., 98, 9215-9231, 1993.

Feldstein, Y.I., Modeling of the magnetic field of magnetospheric ring current as a function of interplanetary medium parameters, Space Sci. Rev., 59, 1992.

Gonzalez, W. D., B. T. Tsurutani, A. L. C. Gonzalez, E. J. Smith, F. Tang, and S. I. Akasofu, Solar wind-magnetosphere coupling during intense magnetic storms (1978-1979), J. Geophys. Res., 94, 8835-8851, 1989.

Mayaud, P. N., Derivation, meaning and use of geomagnetic indices, $A G U$ monograph, Washington DC, 1980.

Mendes Jr., O., The interplanetary origin and the main-phase development of moderate geomagnetic storms, PhD Thesis, INPE, São José dos Campos, Brazil, 1992.

Rostoker, G., Geomagnetic indices, Rev. Geophys. Space Phys., 10, 935-950, 1972.

Rostoker, G., A quantitative relationship between $\mathrm{AE}$ and Kp, $J$. Geophys. Res., 96, 5853-5857, 1991.

Russell, C. T., R. L. McPherron, Semiannual variation of geomagnetic activity, J. Geophys. Res., 78, 1973.

Tsurutani, B. T., W. D. Gonzalez, F. Tang, S. I. Akasofu, and E. J. Smith, Origin of interplanetary southward magnetic fields responsible for major magnetic storms near solar maximum (1978-1979), J. Geophys. Res., 93, 8519-8531, 1988.

Tsurutani, B. T., W. D. Gonzalez, A. L. C. Gonzalez, F. Tang, J. K. Arballo, and M. Okada, Interplanetary origin of geomagnetic activity near solar minimum (1973-1975), J. Geophys. Res., 100, 21717-21733, 1995. 